\section{Amostragem em pesquisas qualitativas: proposta de procedimentos para constatar saturação teórica}

\author{
Sampling in qualitative research: a proposal for \\ procedures to detect theoretical saturation
}

\footnotetext{
1 Centro de Ciências Biológicas e da Saúde, Universidade Federal de São Carlos, São Carlos, Brasil. 2 Faculdade de Medicina Universidade Federal de Minas Gerais, Belo Horizonte, Brasil.

3 Faculdade de Ciências Médicas, Universidade Estadual de Campinas, Campinas, Brasil.

Correspondência B. J. B. Fontanella Departamento de Medicina, Centro de Ciências Biológicas e da Saúde, Universidade Federal de São Carlos. Rodovia Washington Luís km 235, SP 310, São Carlos, SP 13565-905, Brasil. bruno@ufscar.br
}

\begin{abstract}
A qualitative study's methodological transparency is considered a key factor for achieving its reliability and should be guaranteed by the researchers. Closing the sampling process by saturation is a common approach, but it is rarely made explicit in research reports. Qualitative researchers also commonly experience technical difficulties in objectively identifying saturation. This article proposes a method to organize sample closing by saturation, with a sequence of eight procedural steps for treating and analyzing data collected through open or semi-structured interviews or focus groups. The article aims to help researchers objectively explain how and when saturation occurred and to allow their readers to know how this process occurred.
\end{abstract}

Sampling Studies; Sample Size; Qualitative Research; Methods
Bruno Jose Barcellos Fontanella ${ }^{1}$

Bruna Moretti Luchesi 1

Maria Giovana Borges Saidel 1

Janete Ricas 2

Egberto Ribeiro Turato 3

Débora Gusmão Melo 1

\section{Introdução}

Ser transparente quanto à técnica de amostragem utilizada é uma atitude da dimensão ética que ajuda a evidenciar o rigor empregado em uma investigação científica. Propomos aqui um modo de sistematizar e de expor o tratamento e análise de dados coletados em pesquisas qualitativas que utilizam as chamadas amostras fechadas por saturação teórica.

"Fechar" a amostra significa definir o conjunto que subsidiará a análise e interpretação dos dados. Nas amostras não-probabilísticas (intencionais), tal definição é feita a partir da experiência do pesquisador no campo de pesquisa, numa empiria pautada em raciocínios instruídos por conhecimentos teóricos da relação entre o objeto de estudo e o corpus a ser estudado 1 .

Se não houve fechamento por exaustão (abordando todos os sujeitos elegíveis), deve-se justificar por que se interrompeu o processamento de novas observações e o recrutamento de novos participantes. Uma das maneiras de fazê-lo corresponde ao processo de amostragem por saturação teórica: interrompe-se a coleta de dados quando se constata que elementos novos para subsidiar a teorização almejada (ou possível naquelas circunstâncias) não são mais depreendidos a partir do campo de observação 1,2 .

Uma pesquisa decorre de questões, sendo então impossível prescindir de estabelecer quais dados são necessários e suficientes para respon- 
dê-las. Considera-se haver saturação empírica quando o pesquisador constata deles dispor $1 \mathrm{e}$ que há saturação teórica quando a interação entre campo de pesquisa e o investigador não mais fornece elementos para balizar ou aprofundar a teorização 1,2 .

Algumas questões conceituais e metodológicas sobre saturação foram anteriormente comentadas em texto que chamou a atenção para o risco de uma excessiva subjetividade nos critérios empregados para a finalização do recrutamento de participantes ${ }^{3}$. No presente artigo, o objetivo é aprofundar uma proposta, resumida na Tabela 1 , de uma técnica de tratamento de dados que permita aos leitores de relatórios de pesquisas conhecerem de que modo foi constatada a saturação teórica.

\section{Passos procedimentais sugeridos}

Passo 1: disponibilizar os registros de dados "brutos"

De início, recomendamos que, além das transcrições integrais dos diálogos gravados, sejam também disponibilizados arquivos digitais com as correspondentes gravações de áudio. Dispor de ambos facilita um processo pormenorizado de interpretação, pois o suporte auditivo permite perceber elementos de entonação e prosódia e as transcrições possibilitam observações mais reflexivas e detidas sobre alguns trechos.

\section{Passo 2: "imergir" em cada registro}

Os pesquisadores "exploram" individualmente cada uma das entrevistas, à medida que estejam disponíveis. Caso se esteja trabalhando com pré-categorias analíticas, os temas e enunciados correspondentes, que cada pesquisador considere importantes para instruí-las, são anotados. Se forem previstas formulações de novas categorias, cada pesquisador também as registra para depois discuti-las com os pares.

\section{Passo 3: compilar as análises individuais} (de cada pesquisador, para cada entrevista)

Um dos pesquisadores compila os temas e tipos de enunciados identificados por ele próprio e pelos outros investigadores. Caso sejam sugeridas novas categorias analíticas, o compilador poderá agrupá-las, pois os colaboradores podem dar nomes diferentes a conceitos e idéias muito semelhantes. Numa reunião presencial as divergências nas análises individuais são discutidas em busca do consenso.
Para facilitar essa compilação, sugerimos que os parágrafos das transcrições sejam identificados, um a um, facilitando o manejo desses trechos, podendo os pesquisadores identificar, imediatamente, o participante e o momento da entrevista em que foram proferidos.

\section{Passo 4: reunir os temas ou tipos de enunciados para cada pré-categoria ou nova categoria}

Para cada categoria são agrupados os correspondentes temas e tipos de enunciados identificados e consensuados, fazendo constar os respectivos trechos das entrevistas que os exemplificam.

\section{Passo 5: codificar ou nominar os dados}

A nominação deve representar o conjunto de idéias, valores, sentimentos etc., abarcando as manifestações dos entrevistados que contribuem para compreender melhor o que expressaram. Aqui se concentra parte do trabalho criativo de pesquisa, fundamentado em conhecimentos e envolvendo raciocínios indutivos: parte-se do particular (um ou vários enunciados) e chega-se a algo mais abstrato e geral (a nominação deste conjunto). Nominar algo que anteriormente não tinha nome revela as inclinações teórico-ideológicas dos pesquisadores, podendo este procedimento ser revisto continuamente, de acordo com os aprofundamentos conceituais que se processem no estudo adicional e na discussão. Não se trata de vincular imediatamente a denominação escolhida a determinada teoria. Embora se considere que nomear seja uma atitude expressiva do pensamento, reveladora dos conhecimentos de quem nomeia, mesmo que o pesquisador conheça profundamente certas teorias, poderá mantê-las temporariamente em suspensão (atitude próxima à epoché dos fenomenologistas) para evitar uma possível ilusão de transparência do material empírico ${ }^{4}$.

\section{Passo 6: alocar (numa tabela) os temas e tipos de enunciados}

Trata-se de uma alocação que permita a visualização dos elementos analíticos com que se trabalha.

A Tabela 2 é um exemplo hipotético de alocação de dados, nela constando ficticiamente o registro de quais foram os entrevistados que proferiram determinado tipo de enunciado ou tema a respeito da pré-categoria "quadro clínico". Seu propósito principal é permitir uma visualização de como se distribuíram os 11 tipos de enunciados identificados ao longo das 12 entrevistas. 
Passos procedimentais para constatação da saturação teórica.

\begin{tabular}{|c|c|}
\hline Passos & Descrição \\
\hline $\begin{array}{l}\text { Passo 1: disponibilizar os registros de dados } \\
\text { "brutos" }\end{array}$ & $\begin{array}{l}\text { Todos os pesquisadores têm acesso aos registros integrais de áudio e } \\
\qquad \text { aos transcritos, desde as primeiras coletas }\end{array}$ \\
\hline Passo 2: "imergir" em cada registro & $\begin{array}{l}\text { São feitas leituras e audições individuais, visando a identificar núcleos } \\
\text { de sentido nas manifestações dos sujeitos participantes }\end{array}$ \\
\hline $\begin{array}{l}\text { Passo 3: compilar as análises individuais (de } \\
\text { cada pesquisador, para cada entrevista) }\end{array}$ & $\begin{array}{c}\text { Aqui, as entrevistas são o foco: compilar os temas e tipos de } \\
\text { enunciados identificados em cada uma delas, por cada um dos } \\
\text { pesquisadores do grupo (instruindo as pré-categorias ou as eventuais } \\
\text { novas categorias) }\end{array}$ \\
\hline $\begin{array}{l}\text { Passo 4: reunir os temas ou tipos de } \\
\text { enunciados para cada pré-categoria ou nova } \\
\text { categoria }\end{array}$ & $\begin{array}{l}\text { Aqui, os temas ou tipos de enunciados são o foco: depois de cada } \\
\text { entrevista ser analisada pelos pesquisadores, agregam-se as falas } \\
\text { consideradas exemplares dos núcleos de sentido identificados }\end{array}$ \\
\hline Passo 5: codificar ou nominar os dados & $\begin{array}{c}\text { Nominação dos temas e dos tipos de enunciados contidos em cada } \\
\text { pré-categoria ou nova categoria }\end{array}$ \\
\hline $\begin{array}{l}\text { Passo 6: alocar (numa tabela) os temas e tipos } \\
\text { de enunciados }\end{array}$ & $\begin{array}{l}\text { Agregando-os para cada (pré-) categoria e destacando quando se } \\
\text { deu a primeira ocorrência }\end{array}$ \\
\hline $\begin{array}{l}\text { Passo 7: constatar a saturação teórica para } \\
\text { cada pré-categoria ou nova categoria }\end{array}$ & $\begin{array}{l}\text { Isso ocorre quando novos temas ou tipos de enunciados não são, de } \\
\text { maneira consistente, acrescentados após novas entrevistas }\end{array}$ \\
\hline Passo 8: "visualizar" a saturação & $\begin{array}{l}\text { Transformação da tabela em um gráfico, possibilitando, para cada } \\
\text { categoria analisada, uma constatação visual da "saturação" }\end{array}$ \\
\hline
\end{tabular}

Tabela 2

Exemplo hipotético da distribuição de frequência de enunciados referentes a uma categoria de análise fictícia ("quadro clínico") *.

\begin{tabular}{|c|c|c|c|c|c|c|c|c|c|c|c|c|c|}
\hline \multirow[t]{2}{*}{ Tipos de enunciados } & \multicolumn{12}{|c|}{ Entrevistas } & \multirow[t]{2}{*}{ Total de recorrências } \\
\hline & 1 & 2 & 3 & 4 & 5 & 6 & 7 & 8 & 9 & 10 & 11 & 12 & \\
\hline Diversidade biológica & $\mathrm{x}$ & $\mathrm{x}$ & & $\mathrm{x}$ & & $\mathrm{x}$ & & $\mathrm{x}$ & $\mathrm{x}$ & & & & 6 \\
\hline Diversidade do quadro clínico em relação ao sintoma y & $\mathrm{x}$ & & & & & & & & $x$ & & & & 2 \\
\hline Menções a problemas que não fazem parte do sintoma y & $\mathrm{x}$ & & $x$ & $x$ & & $x$ & & & & & $x$ & & 5 \\
\hline Diferentes gravidades de acometimento & & $\mathrm{x}$ & & & & & & $\mathrm{x}$ & & $x$ & & $x$ & 4 \\
\hline Sinal ké patognomônico & & $x$ & & & & & & & & $x$ & $x$ & & 3 \\
\hline Sinal w é fator de exclusão & & & $\mathrm{x}$ & & $x$ & & & & & & & $x$ & 3 \\
\hline Não conhece a semiotécnica para o sinal z & & & $x$ & & & & & & & & & & 1 \\
\hline Papel de exames complementares & & & & & $\mathrm{x}$ & & $x$ & & $x$ & & & & 3 \\
\hline Desvalorização da semiotécnica & & & & & $x$ & & & & & & & & 1 \\
\hline Diversidade psicossocial do quadro clínico & & & & & $x$ & $x$ & $x$ & $x$ & & & & $x$ & 5 \\
\hline Insegurança em relação ao quadro clínico & & & & & & $x$ & & & & & $x$ & $x$ & 3 \\
\hline Total de novos tipos de enunciados para cada entrevista & 3 & 2 & 2 & 0 & 3 & 1 & 0 & 0 & 0 & 0 & 0 & 0 & - \\
\hline
\end{tabular}

x: recorrências; $\mathbf{X}$ : novo tipo de enunciado.

* Para esta categoria ("quadro clínico"), em específico, a saturação foi constatada como tendo ocorrido na sexta entrevista.

Observe (na soma das recorrências, na última coluna à direita) que alguns enunciados apareceram uma única vez, mas outros são frequentes. O número de recorrências não é importante per se, pois apenas revela o que teria sido mais comumente enunciado em uma amostra intencional, refletindo históricos pessoais específicos, sem possibilidades de generalização matematizada para o universo estudado. Entretanto, o pesquisador talvez queira discutir com 
mais ênfase esses dados, pressupondo que sejam parte importante do imaginário do grupo ou considerando tratar-se de temas com maiores possibilidades de exemplificação, mais aderidos à empiria. Nessa lógica, "diversidade biológica" mereceria particular atenção na discussão. Porém, mesmo os enunciados ou temas que apareceram uma única vez certamente devem ser discutidos, pois podem ser produtos de visões mais abrangentes ou profundas de um participante em particular.

\section{Passo 7: constatar a saturação teórica para cada pré-categoria ou nova categoria}

Se a quantificação das recorrências não é importante, uma outra nos parece ser: a soma, na última linha, da frequência de aparecimento de novos temas ou tipos de enunciados, em cada entrevista. Essa soma pode ser útil para uma constatação mais objetiva do processo de saturação.

No exemplo representado pela Tabela 2, os novos tipos de enunciados tenderam a se concentrar nas três primeiras entrevistas, não havendo novas ocorrências nas seis últimas. Uma ocorrência na sexta entrevista encerrou essa sequência. Observe que a "queda" ocorreu de maneira nítida. Isso aconteceu a despeito de as primeiras entrevistas terem possibilitado "calibrar" o entrevistador em processo, permitindo que ele fizesse uma sondagem progressivamente mais qualificada, capacitando-o e qualificando-o para melhor perscrutar os entrevistados seguintes.

A Tabela 2 mostra como a dinâmica de tratamento e análise dos dados terminaram por levar à interrupção da captação de novos participantes: constatou-se o escasseamento de novos tipos de enunciados, correspondendo ao que se designa como saturação teórica (foi atingido, supostamente, o adensamento teórico possível, com base nos dados empíricos disponíveis e nos atributos analíticos e interpretativos dos pesquisadores) 2,3. A coleta de novos dados por meio de novas entrevistas acrescentaria supostamente poucos elementos para discussão em relação à densidade teórica já obtida.

O momento em que começam a escassear novos tipos de enunciados ou temas, para depois “desaparecerem”, não se dá exatamente na mesma entrevista para cada categoria analisada. Para a categoria representada na Tabela 2, a saturação teórica ocorreu na sexta entrevista. Para uma outra, isso poderia acontecer na terceira ou na nona, por exemplo.

\section{Passo 8: "visualizar" a saturação}

A Figura 1 mostra com maior clareza visual a ideia expressa no parágrafo anterior, mostrando como se distribuiu a ocorrência de novos enunciados para três categorias: "quadro clínico", "diagnóstico" e "tratamento”. Para "quadro clínico”, como já comentado, houve numerosos enunciados novos no início da coleta de dados, mas após a sexta entrevista isto não ocorreu mais (= saturação constatada para esta categoria).

Entretanto, as entrevistas continuaram porque as outras categorias ("diagnóstico" e "tratamento") ainda não haviam atingido esse ponto. Nesse exemplo, a saturação teórica geral foi con-

Figura 1

Visualização da "dinâmica de saturação" de três categorias distintas (exemplo hipotético) *
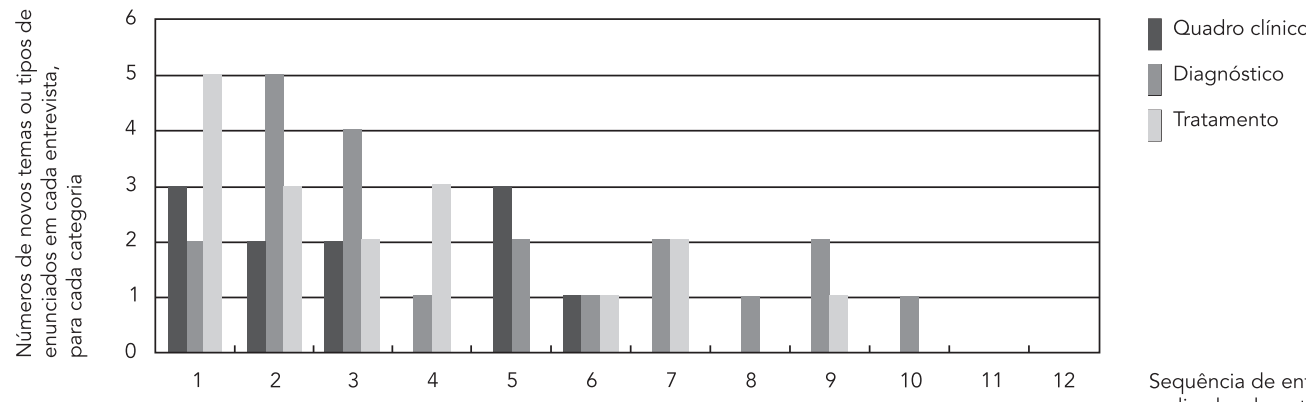

Tratamento

Sequência de entrevistas realizadas durante a pesquisa

\footnotetext{
* Neste exemplo, constatou-se (sempre retrospectivamente) que a categoria "quadro clínico" foi saturada teoricamente na sexta entrevista; a categoria "tratamento" na nona e a categoria "diagnóstico" na décima.
} 
siderada retrospectivamente como tendo acontecido na décima entrevista, tendo a última entrevista sido realizada com o intuito de reforçar esta percepção.

\section{Conclusões}

Buscar compreender fenômenos subjetivos (significados culturais e/ou psicológicos) não significa adotar, como por vezes se observa, uma postura "subjetivista", que chega a desconsiderar triviais aspectos metodológicos e procedimentais sob o argumento de serem meras preocupações positivistas ${ }^{5}$.

Neste texto, discutimos como se pode proceder para efetivar e demonstrar algo que é inevitável também nas pesquisas qualitativas: estabelecer o tamanho final da amostra. A técnica sugerida contém procedimentos trabalhosos (tanto quanto ocorre na organização e tratamento de dados nas pesquisas quantitativas), mas pode ser adaptada às especificidades de cada trabalho.

\section{Resumo}

A transparência metodológica na pesquisa qualitativa é fator que contribui para sua confiabilidade, devendo ser garantida pelos pesquisadores. A utilização do critério de fechamento amostral por saturação é freqüente, entretanto a maneira como este processo ocorreu é raramente explicitado nos relatórios de pesquisa. Dificuldades técnicas para uma constatação objetiva da saturação também são comuns entre os pesquisadores qualitativos. Neste texto, propõe-se um modo prático de organizar o trabalho de fechamento amostral por saturação, expondo uma seqüência de oito passos procedimentais de tratamento e análise de dados coletados em entrevistas não-dirigidas com questões abertas ou em grupos focais. Almeja-se contribuir para que os pesquisadores explicitem objetivamente o modo como a saturação ocorreu e, aos seus leitores, conhecer como se deu este processo.

Amostragem; Tamanho da Amostra; Pesquisa Qualitativa; Métodos
Um tratamento adequado dos significados atribuídos ao processo saúde/doença é de particular interesse para quem se dedica a estudar aspectos da promoção, prevenção e assistência à saúde considerando as perspectivas dos sujeitos implicados. Tal perspectiva é realçada no atual momento do sistema de saúde pública brasileiro, quando se procura garantir a universalização do cuidado e abranger grupos populacionais desprovidos até o momento de uma atenção sistemática. Porém, em qualquer momento histórico-cultural, a área da saúde precisará conhecer as representações e experiências subjetivas de usuários e profissionais, pois sempre se defrontará com diversidades socioculturais, dinâmicas epidemiológicas e mudanças tecnológicas cujas repercussões subjetivas, individuais ou coletivas merecerão ser compreendidas. Portanto, pesquisas qualitativas em saúde precisarão ser feitas continuamente, na tentativa de captar e explorar essa realidade empírica processual, sempre em construção 4 , cabendo aos pesquisadores procurar zelar pelo rigor científico dessas produções.

\section{Colaboradores}

B. J. B. Fontanella e D. G. Melo conceberam e planejaram o artigo, elaboraram o rascunho e organizaram as demais versões do texto ora apresentado, e aprovaram a versão final do manuscrito. B. M. Luchesi, M. G. B. Saidel, J. Ricas e E. R. Turato contribuíram significativamente na elaboração do rascunho e na revisão crítica do conteúdo, e participaram da aprovação da versão final do manuscrito. 


\section{Referências}

1. Pires AP. Amostragem e pesquisa qualitativa: ensaio teórico e metodológico. In: Poupart J, Deslauriers JP, Groulx LH, Lapemère A, Mayer R, Pires AP, organizadores. A pesquisa qualitativa: enfoques epistemológicos e metodológicos. Petrópolis: Editora Vozes; 2008. p. 154-211.

2. Glaser G, Strauss A. The discovery of grounded theory: strategies for qualitative research. New York: Aldine de Gruyter; 1967.

3. Fontanella BJB, Ricas J, Turato ER. Amostragem por saturação em pesquisas qualitativas em saúde: contribuições teóricas. Cad Saúde Pública 2008; 24:17-27.

4. Minayo MCDS. O desafio do conhecimento: pesquisa qualitativa em saúde. 10a Ed. São Paulo: Editora Hucitec; 2007.

5. Gobo G. Sampling, representativeness and generalizability. In: Seale C, Gobo G, Gubrium JF, Silverman D, editors. Qualitative research practice. London/Thousand Oaks/New Delhi: Sage Publications; 2007. p. 405-26.

Recebido em 10/Ago/2010

Versão final reapresentada em 11/Out/2010

Aprovado em 26/Out/2010 